Research Paper

\title{
Three-dimensional Ultrasound Appearance of Pelvic Floor in Nulliparous Women and Postpartum Women One Week after Their First Delivery
}

\author{
Feifei Liu, Lian $\mathrm{Xu}$, Tao Ying ${ }^{\bowtie}$, Junjia Tao, and Bing $\mathrm{Hu}$ \\ Department of Ultrasound in Medicine, Shanghai Jiao Tong University Affiliated Sixth People's Hospital; Shanghai Institute of Ultrasound \\ in Medicine, Shanghai, People's Republic of China.
}

$\triangle$ Corresponding author: Tao Ying, PhD, Department of Ultrasound in Medicine, Shanghai Jiao, Tong University Affiliated Sixth People's Hospital, Yishan Road 600 \#, Shanghai 200233, People's Republic of China. E-mail: yingtaomail@yeah.net, qaz1988421@sjtu.edu.cn; Phone: 0086-2164369181- 8877; Fax: 0086-21-54488254

(C) Ivyspring International Publisher. This is an open-access article distributed under the terms of the Creative Commons License (http://creativecommons.org/ licenses/by-nc-nd/3.0/). Reproduction is permitted for personal, noncommercial use, provided that the article is in whole, unmodified, and properly cited.

Received: 2013.08.12; Accepted: 2013.12.II; Published: 20I4.0I.II

\begin{abstract}
This study investigated the morphology and structure of pelvic floor in 50 nulliparous and 95 postpartum women (47 vaginal delivery, 48 Cesarean section) using translabial three-dimensional (3D) ultrasound. All the primiparae underwent ultrasound examination within one week after their first delivery. Volume datasets were acquired and analyzed to determine the alterations of levator hiatus after childbirth. Significant differences were observed in the levator hiatus of postpartum women compared with that of nullipara women. In postpartum women, the levator hiatus, with their dimensions increased, expanded into a circular shape. Puborectalis was avulsed in eight cases (accounting for $8.42 \%$ of all) and pelvic organ prolapse was found in 12 cases (accounting for $12.63 \%$ ). The hiatal dimensions were larger and the incidence of pubrectalis muscle avulsion (17.02\% vs. $0 \%)$ and pelvic organ prolapse (21.28\% vs. $4.17 \%)$ was significantly higher in Vaginal delivery group than Cesarean section group. In summary, 3D ultrasound is an effective tool to detect the pelvic floor of postpartum women who present with morphological abnormalities, and such abnormalities are more likely to show in vaginal delivery women compared to Cesarean section.
\end{abstract}

Key words: Levator hiatus; Pelvic floor; Postpartum women; Delivery; Three-dimensional ultrasound.

\section{Introduction}

Pelvic floor dysfunction (PFD) encompasses a number of highly prevalent clinical conditions such as female pelvic organ prolapse, urinary and fecal incontinence, and sexual dysfunction ${ }^{[1]}$. Of those diseases, pelvic organ prolapse is relatively more common, with a mean prevalence of about $19.7 \%$ (range: $3.4-56.4 \%)$ in developing countries ${ }^{2]}$. Although there is a general lack of data regarding the etiology of female pelvic organ prolapse, its association with childbirth is well established. Current evidence suggests that levator trauma constitutes at least a part of the 'missing link' between delivery and female pelvic organ prolapse ${ }^{[3]}$. Other factors include denervation of pelvic floor muscles ${ }^{[4]}$, damage to fascia, connective tissue remolding ${ }^{[5]}$, and hormonal changes in peripartum may also play a role in some individuals.

Morphological changes of levator hiatus during pregnancy and delivery may have clinical significance in the subsequent development of PFD. Childbirth results in increased distensibility of the levator hiatus, and the enlargement of hiatus is more obvious especially after levator ani avulsion. A population-based study reported that women undergoing either vaginal delivery or Cesarean section had a greater risk of developing pelvic floor dysfunctions than nulliparous women. Alterations in levator biometry, structure and 
function are also associated with the mode of delivery. Women with vaginal or operative vaginal delivery had a significantly larger hiatal area and transverse diameter than women who delivered by Cesarean section ${ }^{[6]}$. Vaginal delivery is associated with an increased rate of avulsion of puborectalis component of the levator ani muscle, and the use of forceps or vacuum also increases the damage ${ }^{[7-8]}$. A lower pelvic floor muscle strength was observed in women with a history of vaginal delivery compared to those undergoing Cesarean section[9-10]. In addition, recent studies showed that childbirth-related trauma may involve not only the levator ani muscle but also damage to fascia and connective tissue ${ }^{[11-12]}$.

Alterations of the levator hiatus morphology following delivery have been demonstrated using magnetic resonance imaging (MRI) and more recently, three-dimensional (3D) ultrasound imaging [13-16]. This study utilized 3D ultrasonography to assess the morphological alterations of levator hiatus in women undergoing vaginal delivery and Cesarean section one week after their first delivery and compared it with nulliparous female, aiming to provide imaging basis for the study of relationship between delivery and PFD.

\section{Materials and Methods}

\section{Subjects}

This study was undertaken in Shanghai Jiao Tong University Affiliated Sixth People's Hospital where 2800 deliveries took place per year. The female who gave birth between July 2012 and February 2013 in the Department of obstetrics were recruited into the postpartum group. Inclusion criteria were an uncomplicated singleton pregnancy, at 37-42 weeks' gestation and first delivery. Women who developed pathology during the pregnancy were excluded (such as gestational hypertension, diabetes, intrauterine growth restriction and PFD). These women were divided into two groups based on their type of delivery: Vaginal delivery group and Cesarean section group.
All of the vaginal primipara showed cephalic presentation and had a spontaneous vaginal delivery without instrument-assisted; Cesarean sections were planned pre-labor. Recruitment of the controls was primarily from the normal nullipara who came to the department of Gynecology because of irregular menstruation, colpitis, pelvic inflammatory disease, and so on. Those with a history of miscarriage at 16 weeks, previous PFD or pelvic floor surgery, pelvic tumor or chronic cough were excluded. Recruitment continued until a minimum of 50 patients per group was reached. A total of 100 postpartum women (Postpartum group) and 50 nulliparas (Nullipara group) were recruited from July 2012 to February 2013. Local Ethics Committee approval was obtained. All women gave their informed consent before being enrolled in this study.

\section{Ultrasound examination}

Imaging was performed using GE Voluson E8 (GE Medical Systems, Zipf, Austria) with RAB4-8-D $3 \mathrm{D}$ volume probe. The probe was covered with a glove and placed on the perineum in the sagittal plane. Volume datasets (at rest, on maximum Valsalva and maximum pelvic floor contraction) were acquired in supine position after voiding, and were processed offline using 4D View software Version 1 (GE Medical systems). The morphology and structure of levator hiatus (the hiatal dimensions, puborectalis and pelvic organs) were observed and compared between groups. All ultrasound volumes of postpartum women were acquired at 5-7 days (in 1 week) after delivery by two experienced sonographers.

Figure 1 demonstrates the identification of the minimum plane of levator hiatus, in both midsagittal and oblique axial plane ${ }^{[17]}$. Figure 2 shows the morphological parameters on the minimum plane measured by one experienced investigator: HA (levator hiatus area), AP (anteroposterior diameter of levator hiatus) and LR (transverse diameter of levator hiatus from left to right).
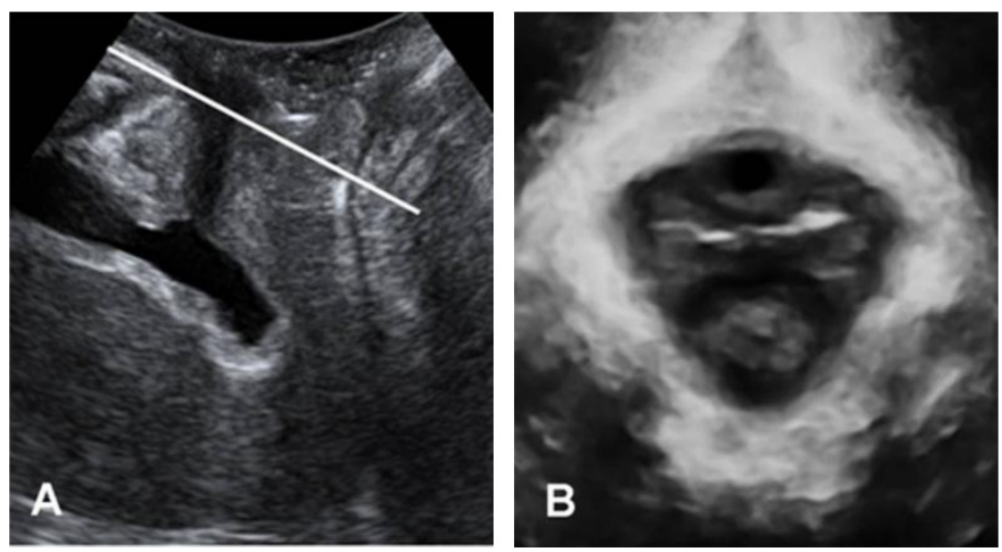

Figure I. The plane of minimal hiatal dimensions: mid-sagittal plane $(A)$ and axial plane (B). 


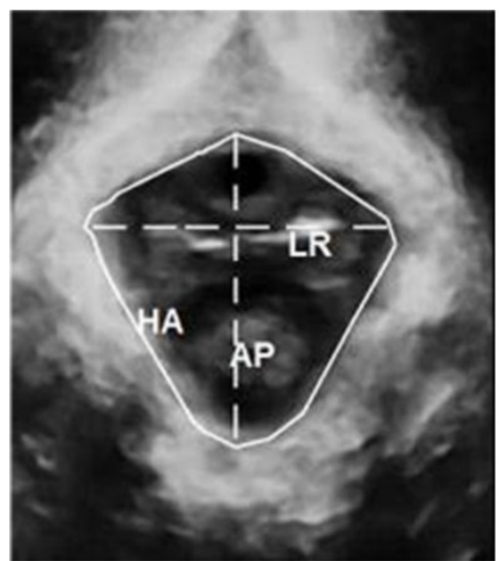

Figure 2. Measurement of levator hiatus in the minimal hiatal dimensions: HA (hiatal area), AP (anteroposterior diameter), and LR (transverse diameter of levator hiatus from left to right).

The diagnosis of puborectalis avulsion was made on tomographic ultrasound imaging (TUI) on the maximum pelvic floor contraction. A set of 8 parallel tomographic slices was obtained in the axial plane at intervals of $2.5 \mathrm{~mm}$ from $5.0 \mathrm{~mm}$ caudal to $12.5 \mathrm{~mm}$ cephalad of the plane of minimal hiatal dimensions. The puborectalis avulsion was diagnosed when there was loss of continuity between muscle and pelvic sidewall at least on one slice. Avulsion includes full avulsion and partial avulsion. Full avulsion is determined if the loss of continuity between muscle and pelvic sidewall is seen on all the three central slices; at the plane of minimal hiatal dimensions and two slices above it ${ }^{[18]}$. Partial avulsion is diagnosed when loss of continuity between muscle and pelvic sidewall is seen in less than the three central slices of TUI or in other planes than these slices.

Two-dimensional (2D) ultrasound were used to determine pelvic organ prolapse in midsagittal plane on maximal Valsalva maneuver (best of at least three maneuvers) ${ }^{[19]}$. The inferiorposterior margin of the symphysis pubis serves as a line of reference. On maximum Valsalva, the maximum descent of the bladder, the cervix or vaginal vault, the cul, the sac and rectum were determined relative to the reference line. In normal condition, the leading edge of pelvic organs was above the reference line; otherwise, it was defined as pelvic organ prolapse.

\section{Statistical analysis}

Statistical analysis was performed after normality testing using SPSS 13.0 (SPSS, Chicago, IL, USA). Mean values were compared by Fisher's ANOVA (for more than two groups) or Student's $t$-test (for two groups). $\chi^{2}$ tests were used for categorical variables. A value of $P<0.05$ was considered statistically significant.

\section{Results \\ General data}

Three women in Vaginal delivery group and two primipara in Cesarean section group were excluded from analysis because of their inability to perform a maximum contraction or Valsalva maneuver, leaving 47 datasets in Vaginal delivery group and 48 datasets in Cesarean section group. Satisfactory volume datasets were obtained in all nulliparae and 95 postpartum women.

General demographics of patients in Vaginal delivery group, Cesarean section group and Nullipara group are shown in Table 1 . There is no significant difference in general demographics of the three groups $(P>0.05)$.

Table 2 shows the measured biometric parameters of the three groups. Compared with the Nullipara group, the HA, AP and LR of the levator hiatus in postpartum women were significantly increased at rest, on maximum Valsalva and maximum pelvic floor contraction, and hiatal dimensions in Vaginal delivery group were larger than that in Cesarean section group $(P<0.001)$.

Table I. General information of Vaginal delivery group, Cesarean section group and Nullipara group

\begin{tabular}{|c|c|c|c|c|}
\hline Variables & $\begin{array}{l}\text { Vaginal delivery } \\
\text { Group }(n=47)\end{array}$ & $\begin{array}{l}\text { Cesarean section } \\
\text { Group }(\mathrm{n}=48)\end{array}$ & $\begin{array}{l}\text { Nulliparous group } \\
(\mathrm{n}=50)\end{array}$ & $P$ value \\
\hline $\operatorname{Age}(y)^{*}$ & $26.00(4.71)$ & $26.28(3.97)$ & $24.56(4.55)$ & NS \\
\hline $\operatorname{BMI}\left(\mathrm{kg} / \mathrm{m}^{2}\right)^{*}$ & $23.74(2.58)$ & $24.09(2.24)$ & $22.74(2.29)$ & NS \\
\hline $\operatorname{BMI}\left(\mathrm{kg} / \mathrm{m}^{2}\right)$ before prenancy\# & $20.90(2.69)$ & $21.26(2.08)$ & & NS \\
\hline $\mathrm{BMI}\left(\mathrm{kg} / \mathrm{m}^{2}\right)$ before delivery\# & $26.71(3.47)$ & $27.02(2.71)$ & & NS \\
\hline Birth weight $(\mathrm{g}) \#$ & $3223.13(419.56)$ & $3437.24(581.31)$ & & NS \\
\hline Gestational time (weeks)\# & $39.43(0.86)$ & $39.43(1.86)$ & & NS \\
\hline
\end{tabular}

Values expressed in mean(SD); SD represents standard deviation. * $P$-values obtained by ANOVA. \#P-values obtained by Student's $t$-test. NS, not significant. 
Table 2. Morphological parameters of the levator hiatus in Nullipara group and Postpartum group: group (Gr.) I = Nullipara group, group $2=$ Vaginal delivery goup, group $3=$ Cesarean section group.

\begin{tabular}{|c|c|c|c|c|c|c|c|c|c|c|c|c|c|}
\hline \multirow[t]{3}{*}{ parameter } & \multirow[t]{3}{*}{ Gr. } & \multirow[t]{3}{*}{ Rest } & \multirow[t]{3}{*}{ Contraction } & \multirow[t]{3}{*}{ Valsalva } & \multicolumn{9}{|c|}{$P$} \\
\hline & & & & & \multicolumn{3}{|c|}{ Gr.1 vs Gr.2 } & \multicolumn{3}{|c|}{ Gr.1 vs Gr.3 } & \multicolumn{3}{|c|}{ Gr.2 vs Gr.3 } \\
\hline & & & & & $\bar{R}$ & C & $\mathrm{V}$ & $\mathrm{R}$ & $\mathrm{C}$ & $\mathrm{V}$ & $\mathrm{R}$ & C & $\mathrm{V}$ \\
\hline \multirow[t]{3}{*}{ HA } & 1 & $12.71(2.61)$ & $10.88(3.06)$ & $15.50(3.47)$ & 0.001 & 0.001 & 0.041 & 0.031 & 0.035 & 0.001 & 0.025 & 0.029 & 0.018 \\
\hline & 2 & $19.94(3.76)$ & $16.17(3.33)$ & $23.13(3.51)$ & & & & & & & & & \\
\hline & 3 & $15.52(3.31)$ & $13.08(3.57)$ & $18.61(3.89)$ & & & & & & & & & \\
\hline \multirow[t]{3}{*}{$\mathrm{AP}$} & 1 & $4.31(0.65)$ & $4.01(0.80)$ & $4.57(0.73)$ & 0.001 & 0.001 & 0.018 & 0.027 & 0.015 & 0.014 & 0.011 & 0.041 & 0.006 \\
\hline & 2 & $5.41(0.73)$ & $4.87(0.68)$ & $5.93(0.57)$ & & & & & & & & & \\
\hline & 3 & $4.75(0.68)$ & $4.59(0.71)$ & $5.11(0.82)$ & & & & & & & & & \\
\hline \multirow[t]{3}{*}{ LR } & 1 & $3.87(0.50)$ & $3.64(0.52)$ & $4.12(0.61)$ & 0.001 & 0.001 & 0.025 & 0.034 & 0.018 & 0.012 & 0.026 & 0.013 & 0.009 \\
\hline & 2 & $4.44(0.47)$ & $4.29(0.63)$ & $4.58(0.67)$ & & & & & & & & & \\
\hline & 3 & $4.09(0.43)$ & $3.88(0.66)$ & $4.33(0.72)$ & & & & & & & & & \\
\hline
\end{tabular}

$\mathrm{HA}$, levator hiatus area; AP, anteroposterior diameter of levator hiatus; LR, left to right diameter of levator hiatus. $\mathrm{R}, \mathrm{C}$ and $\mathrm{V}$ represent rest, contraction and valsalva, respectively.

Values expressed in mean(SD)

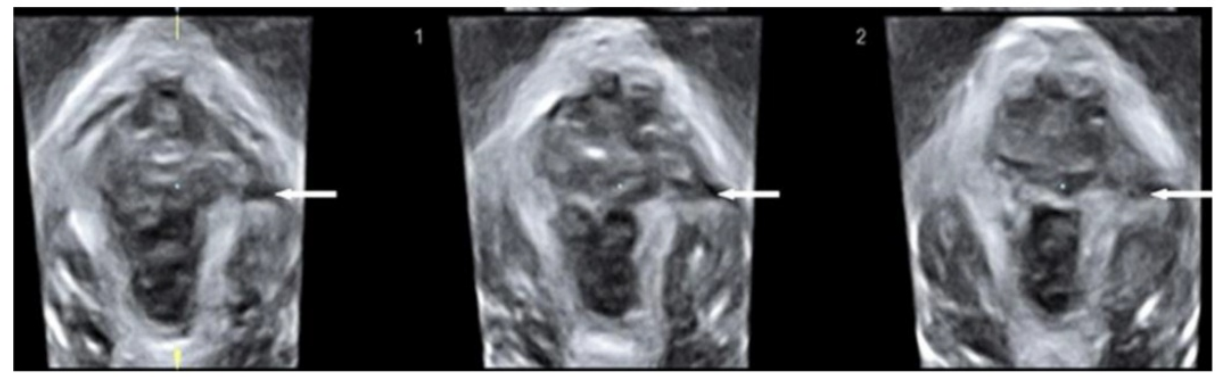

Figure 3. Tomographic ultrasound imaging showing left-sided avulsion of the pubrectalis (arrows pointed).

\section{D ultrasound performance of levator hiatus in Nullipara group}

Levator hiatus was in rhombic shape with compact structure, outlined by puborectalis and pubis. Puborectalis formed a symmetrical and continuous V-shaped sling running from the pelvic sidewall towards the anorectal junction. On the ventral side, puborectalis closely attached to the interior edge of the pubic ramus and surrounded the posterior rectum on the dorsal side. On TUI, the fibers of puborectalis at the site of its insertion were continuous on maximum pelvic floor contraction; avulsion was not detected. On maximum Valsalva, none in Nullipara group was found with pelvic organ prolapse in midsagittal plane. In the axial plane, urethra, vagina and rectum were observed in a line in ventridorsal orientation inside the levator hiatus with clear boundaries.

\section{D ultrasound performance of levator hiatus in Postpartum group}

The levator hiatal dimensions were significantly larger in the postpartum female than that in normal nulliparas, with an oval or circle shape. On TUI, avulsion of the puborectalis muscle was detected in eight cases among those delivered vaginally on maximum pelvic floor contraction (Fig. 3), including 4 unilateral avulsion (2 cases of full avulsion and 2 of partial avulsion) and 4 bilateral avulsion ( 1 case of bilateral full avulsion and 1 case of left-sided full avulsion and right-sided partial avulsion, and 2 cases of right-sided full avulsion and left-sided partial avulsion). No such defects were observed in Cesarean section group. Pelvic organ prolapse was detected on maximum Valsalva. Twelve cases displayed cystocele including 10 from Vaginal delivery group and two from Cesarean section group (Fig. 4). In the axial plane, the arrangement of urethra, vagina and rectum changed, and bladder was also detected in levator hiatus.

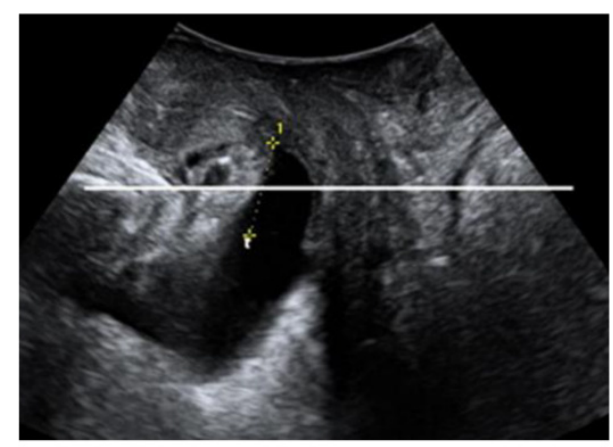

Figure 4. Two dimensional ultrasound imaging showing cystocele. 
The incidence of pelvic organ prolapse $(21.28 \%$ vs. $4.17 \%)$ and pubrectalis muscle avulsion $(17.02 \%$ vs. $0 \%$ ) was significantly higher in Vaginal delivery group compared to Cesarean section group $(P<0.05)$.

\section{Discussion}

Ever since pelvic floor dysfunction disease attracted our attention, pelvic floor assessment and pelvic floor damage have been widely reported or speculated. A majority of women who have given birth are affected by different degrees of pelvic floor trauma. It seems that the assessment of levator hiatus and diagnosis of levator trauma is more repeatable when undertaken with the help of imaging. Magnetic resonance imaging (MRI) once dominated the assessment of pelvic floor; however, it suffers a number of shortcomings such as high cost, restricted accessibility, lack of dynamic imaging ability, unsuitable for women with IUD or cardiac pacemaker. With the emergence of 3D ultrasonography, the study of the levator hiatus has become easier. Rendered 3D volume, with datasets obtained every second, enables visualization of the axial plane, providing good spatial and superior temporal resolution. Apart from that, its low cost, easy accessibility and real-time recording of dynamic changes of pelvic floor makes it ideal for the study of morphological and functional alterations of pelvic floor. Modern 3D ultrasound systems allow tomographic imaging. The diagnosis of levator trauma by TUI is probably the most repeatable technique nowadays, and has been verified to be comparable to MRI [20-21].

This study, using 3D ultrasound, illustrated several morphological alterations in the pelvic floor of postpartum women compared to nulliparas. In the Postpartum group, the hiatal dimensions were larger, the morphology tended to be circular, and puborectalis avulsion and pelvic organ prolapse were also detected.

This study found that the hiatal dimensions in Vaginal delivery group were larger than those in Cesarean section group; the puborectalis avulsion occurred exclusively in Vaginal delivery group; the incidence of pelvic organ prolapse was obviously higher in women who have undergone vaginal delivery.

During delivery, levator ani extended by about $25-245 \%$, allowing the levator hiatus to widen during crowning of the fetal head[22]. Vaginal delivery has been associated with levator trauma, with the assumption being that levator avulsion occurs during crowning of the fetal head[23]. Major levator ani defects are associated with a decrease in pelvic floor muscle contraction strength ${ }^{[24]}$ and are a risk factor for levator hiatus "ballooning", which is the mechanism leading to pelvic organ prolapse[25]. Several studies have investigated that levator avulsion, poor pelvic floor muscle contractility and hiatal ballooning were strong risk factors for recurrence after prolapse repair[26-27].

This study found that the puborectalis avulsion occurred exclusively after vaginal delivery, and the percentage of women with puborectalis avulsion after normal vaginal delivery without instrument-assisted was $17.02 \%$. The result is consistent with previously published data[28-29], which reported an injury rate ranging from $13.3 \%$ to $38.5 \%$ after spontaneous vaginal delivery.

It is assumed that support of the female urethra and bladder is maintained by paraurethral and paravaginal fascial structures, pubourethral ligament and levator ani muscle. Due to inadequate clinical assessment technique or possibly an insufficiently sensitive imaging method, the diagnosis of fascial defects has still been regarded as a challenge. We would like to believe that cystocele occurred in 12 postpartum women may be due to weakening or trauma to either the levator ani muscle or the fasical structures. Further studies in this field will fucous on the imaging appearance of fascial abnormalities.

There are several limitations of our study that should be mentioned. As we did not obtain ultrasound volume datasets prior to childbirth in Postpartum group, peripartum changes in individual patients could not be analyzed. We performed our examinations relatively early in the postpartum period, which means that some of the changes the patients presented may be reversed, such as those due to transient neuropathy; therefore, long-term follow-up observation is underway to establish their true significance and changes of recovery.

\section{Conclusion}

Three-dimensional translabial ultrasound is an effective imaging method for the evaluation of levator hiatus, thanks to its ability of clearly indicating morphological alterations of levator hiatus in early postpartum female, which is of great value to the study of the relationship between delivery and PFD. The process of delivery has an obvious effect on the levator hiatus causing increased levator hiatal dimensions, changes in morphology, puborectalis avulsion or pelvic organ prolapse. Three-dimensional ultrasound shows that vaginal delivery is more likely a major factor for abnormal alterations of levator hiatus.

\section{Acknowledgements}

The Natural Science Foundation of Shanghai funded project is acknowledged for the financial support for this work (13ZR1431300). 


\section{Competing Interests}

The authors have declared that no competing interest exists.

\section{References}

1. Shek KL, Dietz HP. Pelvic floor ultrasonography: an update. Minerva Ginecol 2013; 65: 1-20.

2. Walker GJ, Gunasekera P. Pelvic organ prolapse and incontinence in developing countries: Review of prevalence and risk factors. Int Urogynecol J 2011; 22: 127-35.

3. Dietz HP, Gillespie AV, Phadke P. Avulsion of the pubovisceral muscle associated with large vaginal tear after normal vaginal delivery at term. Aust N Z J Obstet Gynaecol 2007; 47: 341-4.

4. Jóźwik M, Jóźwik M. Partial denervation of the pelvic floor during term vaginal delivery. Int Urogynecol J Pelvic Floor Dysfunct 2001; 12: 81-2.

5. Moalli PA, Shand SH, Zyczynski HM, et al. Remodeling of vaginal connective tissue in patients with prolapse. Obstet Gynecol 2005; 106: 953-63.

6. Falkert A, Endress E, Weigl M, et al. Three-dimensional ultrasound of the pelvic floor 2 days after first delivery: influence of constitutional and obstetric factors. Ultrasound Obstet Gynecol 2010; 35: 583-8.

7. Krofta L, Otcenásek M, Kasíková E, et al. Pubococcygeus-puborectalis trauma after forceps delivery: evaluation of the levator ani muscle with 3D/4D ultrasound. Int Urogynecol J Pelvic Floor Dysfunct 2009; 20: 1175-81.

8. Chan SS, Cheung RY, Yiu AK, et al. Prevalence of levator ani muscle injury in Chinese women after first delivery. Ultrasound Obstet Gynecol 2012; 39: 704-9.

9. Gameiro MO, Sousa VO, Gameiro LF, et al. Comparison of pelvic floor muscle strength evaluations in nulliparous and primiparous women: a prospective study. Clinics (Sao Paulo) 2011; 66: 1389-94.

10. Batista EM, Conde DM, Do Amaral WN, et al. Comparison of pelvic floor muscle strength between women undergoing vaginal delivery, cesarean section, and nulliparae using a perineometer and digital palpation. Gynecol Endocrinol 2011; 27: 910-4.

11. Dietz HP, Steensma AB, Hastings R. Three-dimensional ultrasound imaging of the pelvic floor: the effect of parturition on paravaginal support structures. Ultrasound Obstet Gynecol 2003; 21: 589-95.

12. Garriga J, Shek K, Wong V, et al. Does childbirth alter anterior compartment fascial supports? Neurourol Urodyn 2012; 31: 835-6.

13. DeLancey JO, Kearney R, Chou Q, et al. The appearance of levator ani muscle abnormalities in magnetic resonance images after vaginal delivery. Obstet Gynecol 2003; 101: 46-53.

14. Handa VL, Lockhart ME, Kenton KS, et al. Magnetic resonance assessment of pelvic anatomy and pelvic floor disorders after childbirth. Int Urogynecol J 2009; 20: 133-9.

15. Dietz HP. Pelvic floor trauma in childbirth. Aust N Z J Obstet Gynaecol 2013; 53: 220-30.

16. Dietz HP, Shek KL, Chantarasorn V, et al. Do women notice the effect of childbirth-related pelvic floor trauma? Aust N Z J Obstet Gynaecol 2012; 52: 277-81.

17. Dietz HP, Shek C, Clarke B. Biometry of the pubovisceral muscle and levator hiatus by three-dimensional pelvic floor ultrasound. Ultrasound Obstet Gynecol 2005; 25: 580-5

18. Dietz HP, Bernardo MJ, Kirby A, et al. Minima criteria for the diagnosis of avulsion of the puborectalis muscle by tomographic ultrasound. Int Urogynecol J 2011; 22: 699-704.

19. Dietz HP, Haylen BT, Broome J. Ultrasound in the quantification of female pelvic organ prolapse. Ultrasound Obstet Gynecol 2001; 18: 511-4.

20. Zhuang RR, Song YF, Chen ZQ et al. Levator avulsion using a tomographic ultrasound and magnetic resonance-based model. Am J Obstet Gynecol 2011; 205: 232.e1-8

21. Notten K, Weemhoff M. Diagnosing levator defects on 3D transperineal ultrasound compared to MR imaging. Ultrasound Obstet Gynecol 2012; 40 (S1): 19-20.

22. Svabík K, Shek KL, Dietz HP. How much does the levator hiatus have to stretch during childbirth? BJOG 2009; 116: 1657-62.

23. Blasi I, Fuchs I, D'Amico R, et al. Intrapartum translabial three-dimensional ultrasound visualization of levator trauma. Ultrasound Obstet Gynecol 2011; 37: 88-92.

24. Dietz HP, Shek C. Levator avulsion and grading of pelvic floor muscle strength. Int Urogynecol J Pelvic Floor Dysfunct 2008; 19: 633-6.

25. Dietz HP, Simpson JM. Levator trauma is associated with pelvic organ prolapse. BJOG 2008; 115: 979-84.

26. Model AN, Shek KL, Dietz HP. Levator defects are associated with prolapse after pelvic floor surgery. Eur J Obstet Gynecol Reprod Biol 2010; 153: 220-3.

27. Vakili B, Zheng YT, Loesch $\mathrm{H}$, et al. Levator contraction strength and genital hiatus as risk factors for recurrent pelvic organ prolapse. Am J Obstet Gynecol 2005; 192: 1592-8.

28. Cassadó Garriga J Pessarrodona Isern A, Espuña Pons $M$, et al. Four-dimensional sonographic evaluation of avulsion of the levator ani according to delivery mode. Ultrasound Obstet Gynecol 2011; 38: 701-6.
29. Albrich S, Laterza R, Skala C, et al. Impact of mode of delivery on levator morphology: a prospective observational study with $3 \mathrm{D}$ ultrasound early in the postpartum period. BJOG 2012; 119: 51-61. 